\title{
Nanotheranostics
}

2017; 1(2): 166 -177. doi: 10.7150/ntno.18531

Review

\section{Recent strategies to design vascular theranostic nanoparticles}

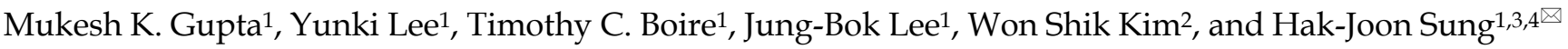 \\ 1. Department of Biomedical Engineering, Vanderbilt University, Nashville, TN, USA; \\ 2. Department of Otorhinolaryngology, Yonsei University, College of Medicine, South Korea; \\ 3. Division of Cardiovascular Medicine, Department of Medicine, Vanderbilt University Medical Center, Nashville, TN, USA; \\ 4. Severance Biomedical Science Institute, College of Medicine, Yonsei University, Seoul, South Korea. \\ $\bowtie$ Corresponding author: hj72sung@yuhs.ac \\ (1) Ivyspring International Publisher. This is an open access article distributed under the terms of the Creative Commons Attribution (CC BY-NC) license \\ (https://creativecommons.org/licenses/by-nc/4.0/). See http://ivyspring.com/terms for full terms and conditions.
}

Received: 2016.11.28; Accepted: 2017.03.11; Published: 2017.04.06

\begin{abstract}
Vascular disease is a leading cause of death and disability worldwide. Current surgical intervention and treatment options for vascular diseases have exhibited limited long-term success, emphasizing the need to develop advanced treatment paradigms for early detection and more effective treatment of dysfunctional cells in a specific blood vessel lesion. Advances in targeted nanoparticles mediating cargo delivery enables more robust prevention, screening, diagnosis, and treatment of vascular disorders. In particular, nanotheranostics integrates diagnostic imaging and therapeutic function into a single agent, and is an emerging platform towards more effective and localized vascular treatment. This review article highlights recent advances and current challenges associated with the utilization of targeted nanoparticles for real-time diagnosis and treatment of vascular diseases. Given recent developments, nanotheranostics offers great potential to serve as an effective platform for targeted, localized, and personalized vascular treatment.
\end{abstract}

Key words: vascular diseases, nanotheranostics

\section{Introduction}

Vascular disease is a leading cause of death in the developed world. According to a 2016 Statistics Update from American Heart Association, one in three Americans dies due to cardiovascular disease [1]. Major vascular diseases include atherosclerosis, aneurysms, stroke, thrombosis, coronary artery disease (CAD), and peripheral artery disease (PAD). Although significant differences exist in terms of the precise nature and location of these disorders, they share many spatiotemporal cellular and molecular mechanisms [2].

Conventional vascular therapies utilize oral, parenteral, intravenous or intra-arterial administration of small molecule drugs and non-preferentially affect both diseased and normal cells. Unwanted systemic side effects and less drug accumulation at target sites occur as a result. Conventional approaches are also limited with respect to rapid drug clearance from circulation and drug instability [3]. Surgical procedures such as angioplasty, stenting, atherectomy, stent-grafting and bypass grafting are also commonly employed [4]. Small molecule drugs have been incorporated into stents, balloon devices, and vascular grafts with marginal improvement in therapeutic efficacy, but these approaches lack imaging and therefore diagnostic capabilities [5]. Non-invasive imaging techniques such as conventional digital subtraction angiography, computed tomography angiography (CTA), magnetic resonance angiography (MRA), and duplex ultrasound are used to aid these approaches [6]. While these imaging modalities have improved diagnostic capabilities, further improvement is needed with respect to over-estimation of stenosis and longer imaging time of calcified arterial stenosis [7]. Therefore, the field of vascular disease requires 
more advanced engineering of therapeutic and imaging modalities [8].

\section{Nanotechnology in medicine}

Recent advances in nanotechnology enables timely detection, prevention and treatment of disease $[9,10]$. These studies are focused toward the development of new methods either for incorporation or encapsulation of therapeutics, imaging agents and targeting ligands to the nanoparticles. In recent years, target-specific engineered nanoparticles have exhibited substantial improvement over conventional treatment options in terms of site-specific localization and reduced side effects [11].

Nanotechnology uses particles with spherical, cylindrical, plate-like and other morphologies in an average size ranging of around ten to several hundred nanometers (nanoparticles) for encapsulation and delivery of small molecule drugs and/or imaging agents. Nanoparticles have inspired considerable research interest as controlled and sustained drug delivery vehicles due to their numerous potential benefits. Desirable characteristics include protection of drugs against degradation via stable encapsulation, ability to deliver poorly soluble drugs, limited toxicity, and site-specific targeting capabilities. Representative nanomaterials include polymeric micelles, nanospheres, and dendrimers, quantum dots, ultra-small superparamagnetic iron oxides (USPIOs), liposomes, and antibodies. These nanoparticles contain surface charge and functional groups which may potentially impact their circulation and macrophage uptake, thereby influencing bio-distribution and delivery of payload at the targeted site. In general, neutral and negatively charged particles have reduced plasma protein adsorption and have a low rate of nonspecific cellular uptake whereas positive charged particles are preferentially uptaken by macrophages [12]. Therefore, nanoparticles can be engineered with an optimum surface charge (e.g. positive, neutral or negative), charge density and targeting ligands in order to extend circulation time, reduce non-specific clearance, and enable effective targeting to a desired location [13].

Most nanomaterial studies have been either focused on development of controlled drug delivery or imaging agents for screening, diagnosis, and therapeutic monitoring. A separate therapeutic delivery or diagnosis-focused nanoparticle approach performs well for some particular functions but is limited with respect to simultaneous real-time monitoring of disease progression and therapeutic treatment. Therefore, multifunctional nanoparticles combined with therapeutic targeting and diagnostic capability is advantageous for real-time disease treatment. Fortunately, extensive research in nanomedicine has afforded the ability to precisely control nanoparticle design features such as size, surface charge and morphology through either the attachment or incorporation of biological and chemical agents within nanoparticles. These versatile nanoparticle features allow for the integrated design, synthesis and fabrication of single agents combined with therapeutic and diagnostic functions [14, 15] (Fig. 1).

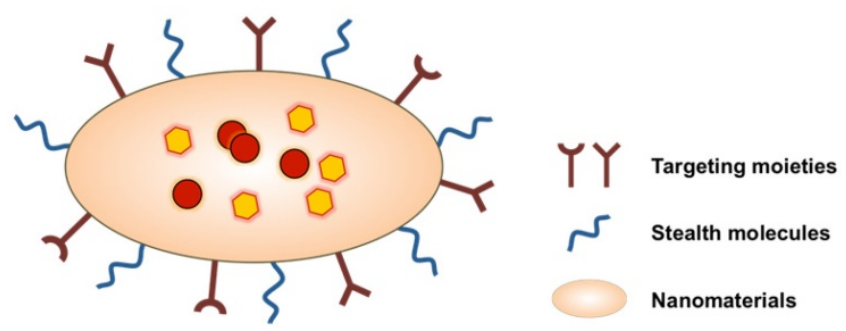

Theranostic nanoparticle

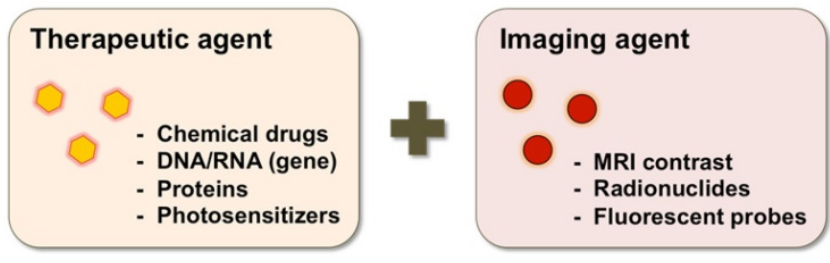

Figure 1. Schematic illustration of theranostic nanoparticles (e.g., micelles, liposomes, nanospheres, dendrimers, quantum dots, iron oxide particles, etc.) embedded with therapeutic and imaging agents.

In 2002, John Funkhouser coined this approach theranostics to describe ongoing efforts to develop such nanoparticle systems for personalized medicine. These multifunctional nanoparticles are called nanotheranostics, which are designed to enable simultaneous disease detection and treatment capabilities [16]. Nanotheranostics as a non-invasive and real-time approach provides a potential therapeutic tool for clinicians to simultaneously monitor drug release at targeted sites and assess its therapeutic function. Studies utilizing theranostic nanoparticles could help physicians to tailor drug doses for the individual patient in a more efficient manner. In this way, therapeutic benefit could be improved through a reduction in toxicity as well as drug costs [17]. Also, development of efficient theranostic vehicles requires an understanding about the underlying disease mechanisms for determining a theranostic agent's targeting location, drug release and therapeutic efficacy [18]. To date, most nanomaterial research is focused on cancer targeting nanotheranostics [19], but research focused on nanotheranostics for vascular diseases such as 
atherosclerosis, thrombosis and cerebrovascular amyloid angiopathy is an emerging field [20]. Several nanoparticle approaches have been investigated either for the treatment or diagnosis of atherosclerosis lesions [21] but this review article mainly focuses on specific approaches for vascular theranostic targeting.

\section{Recent strategies to design vascular theranostic nanomaterials}

Molecular mechanisms of vascular disease have been extensively studied and provide strategies to design specific nanomaterials targeted selectively to diseased cells and tissues. The cellular biomarker specific to endothelial cells (Integrin aV $\beta 3$, CAMS (ICAM, VCAM, and PECAM)), platelets (integrin's GPIIb-IIIa, GPIa-IIa, GPVI, GPIb-IX-V, p-selectin), activated macrophages (scavenger receptors, oxidized LDL receptors) and smooth muscle cells with a pathological, synthetic phenotype can be used as cellular targets for identification of these disease at early stages. Non-cellular biomarkers include lipoproteins, annexins, fibrin, ECM proteins, MMPs, tissue factor, etc. [22]. Nanoparticles can be functionalized with specific ligands/antibodies/ peptides to target the specific vascular regions affected by inflammation, thrombosis, hyperlipidemia or oxidative injury. These research approaches for vascular nanotheranostics have been mostly designed to develop nanoparticles for treatment of atherosclerotic plaques, an underlying cause for most vascular diseases. Theranostic targeted nanoparticles with respect to specific cellular and non-cellular biomarkers are discussed in their respective disease sections.

\section{Atherosclerosis and nanoparticle approaches}

Atherosclerosis is an inflammatory vascular disease characterized by an accumulation of lipid-rich plaques within arteries [23]. Atherosclerosis occurs in arteries for the heart, brain, arms, legs, pelvis, and kidneys and underlies the medical conditions of CAD, aortic aneurysms, PAD, and chronic kidney disease (CKD). Atherosclerosis is initiated through an imbalance in lipid metabolism and a maladaptive immune response that further results in chronic inflammation within the arterial wall. Disease progression starts at an early stage of life that begins with formation of "fatty streaks", which can evolve unstable plaques enriched with macrophages and $\mathrm{T}$ lymphocytes, amongst other cell types. The beginning stage is characterized by adhesion of white blood cells to the activated endothelial monolayer, their directed migration into the intima, maturation of monocytes into macrophages, and formation of foam cells through lipid uptake. The dysfunctional and damaged endothelium further undergoes a series of events for gradual development of atherosclerosis. Progression of the lesion includes the migration of smooth muscle cells (SMCs) to the intima, the proliferation of resident intimal and media-derived SMCs, and over-production of extracellular matrix (ECM) components such as elastin, collagen and proteoglycans. This process results in accumulation of extracellular lipids in advanced lesions through apoptosis of plaque macrophages and SMCs [23].

Macrophages are one of the most important factors in atherosclerotic progression and are known to release inflammatory factors and chemokines that affect healthy blood vessels and thus contribute to neointimal hyperplasia or restenosis following interventional therapies [24]. A vast majority of macrophages in atherosclerotic lesions are produced from local macrophage proliferation instead of inflammatory monocyte recruitment from peripheral blood. Therefore, targeting macrophage proliferation could be a new promising strategy to treat atherosclerosis lesions $[25,26]$.

Photo-induced inflammatory cell ablation using a macrophage-targeted nanoparticle is being used as a therapeutic approach for treatment of atherosclerosis. These nanoparticles are toxic when activated by certain wavelengths of light, thereby reducing off-target side effects. McCarthy et al. developed macrophage-targeting magnetic fluorescence nanoparticles to treat atherosclerosis and in-stent restenosis [27]. In this study, magnetic fluorescent nanoparticles (MFNPs) were prepared by conjugation of $10 \mathrm{kDa}$ dextran-coated magnetic nanoparticles with AF-750 NR dye. In order to impart photodynamic therapy functionality to MFNPs, a photosensitizer, 5-(4-carboxyphenyl)-10,15,20-triphenyl-2,3-dihydroxy chlorin (TPC), was covalently conjugated to the primary amines of MFNP (Fig. 2). MFNPs were stable in DPBS ( $\mathrm{pH} 7.4)$ for months without losing their detection capability through MRI and fluorescence imaging methodologies. Upon excitation of MFNPs at $646 \mathrm{~nm}$, photosensitizer-generated singlet oxygen species specifically killed the macrophages without affecting the neighboring normal cells. The macrophages demonstrated a time-dependent increase in uptake of MFNPs by flow cytometry analysis. In vivo application of these nanoparticles also demonstrated high macrophage uptake, efficient cell killing, and safe monitoring of its spatial distribution by both MRI and near-infrared fluorescence imaging. In another article from the same group, a dextran-coated iron oxide based magneto-fluorescent nanoagent was synthesized by conjugation of 
AlexaFluor 750 and meso-tetraphenylchlorin derivative for near-infrared fluorescent imaging and generation of singlet oxygen, respectively [28]. The theranostic nanoagents were engulfed by phagocytic macrophages and exhibited higher photo toxicity compared to the traditional chlorin derivatives. The efficacy of nanoagents was further tested in an apo $\mathrm{E}^{-/}$mouse animal model. The nanoagents accumulated preferentially inside the atherosclerotic lesions and caused photo-induced apoptosis of phagocytic macrophages [28].

Inflammatory macrophages secrete matrixdisorganizing proteases (e.g., cathepsins and matrix metalloproteinases) at atherosclerotic sites, which can cause thromboembolic stroke or myocardial infarction through atheromata-induced rapture of vulnerable plaques [29]. Initially, Choi et al. investigated the anti-tumor effect of cathepsin B activatable chlorin e6 (Ce6)-functionalized poly-l-lysine grafted with monomethoxy-polyethylene glycol (L-SR15) nanoparticles through protease-mediated photodynamic therapy [30]. Next, an in vitro study from Lee et al. first tested the aforementioned theranostic photodynamic agent for proteolytic cleavage induced death of macrophages upon light illumination by generating highly reactive oxygen species and further studied the effects of atorvastatin and clopidogrel (anti-atherosclerotic drugs) on the effectiveness of photosensitization in cultured murine macrophage RAW 264.7 cells [31]. These drugs didn't influence cellular uptake of L-SR15 but interfered with the in vitro photosensitization effect. In a subsequent study from the same group, Shon et al. tested the diagnostic and therapeutic efficacy of cathepsin-B activatable L-SR15 in a 30 week-old apolipoprotein E knock-out atheromata mouse model for the selective apoptotic attenuation of macrophages and a reduction in catB protease activity [32]. An increase in fluorescence signal from the atheromata region of the L-SR15 group compared to other controls indicated CasB-mediated release of the photosensitizer and preferential accumulation in plaques. L-SR15 didn't cause systemic or neurobehavioral cytotoxicity after photodynamic therapy. Histological images confirmed the selectivity of the L-SR15-based PDT treatment for killing macrophages without affecting smooth muscle cells [32].

5-Aminolevulinic acid (ALA) is an endogenous photodynamic metabolite, which transforms into an active photosensitizer porphyrin IX (PPIX) inside cells through a cellular heme biosynthetic pathway [33]. PPIX is a potent photosensitizer, which can be excited by red light of $635 \mathrm{~nm}$ for generation of singlet oxygen species. ALA-derived PPIX has been shown to accumulate especially in atherosclerotic plaques, and its fluorescence intensity can be positively correlated with the plaque macrophage content [34, 35]. To harness the photodynamic properties of ALA-derived PPIX for early diagnosis and therapy of atherosclerosis, Gonçalves et al., formulated PEGylated ALA gold nanoparticles (ALA:AuNPs)
A)

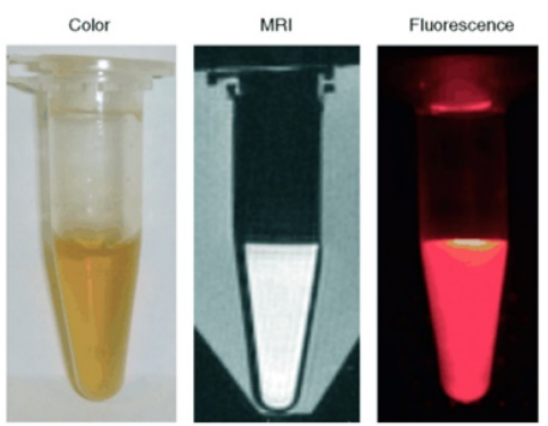

B)
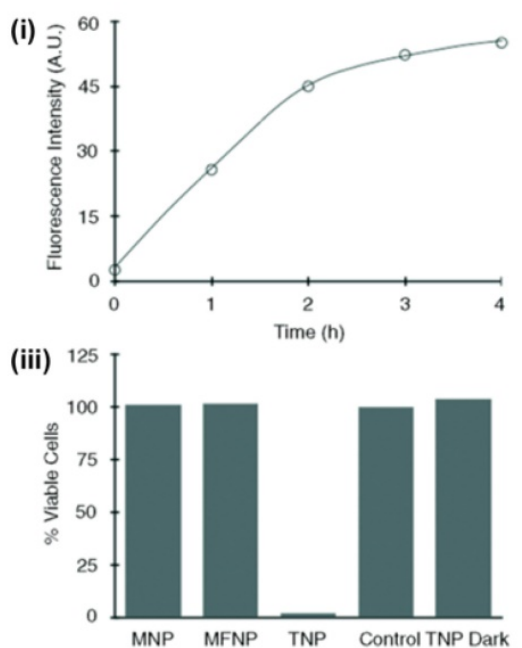

(ii)

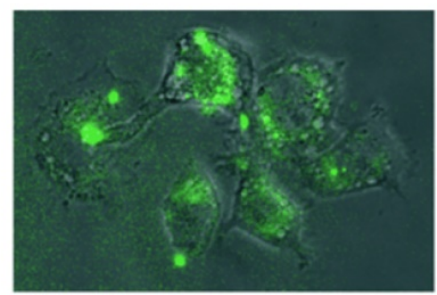

(iv)

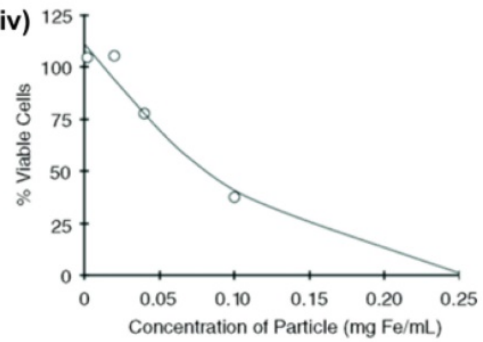

Figure 2. Macrophage-targeted theranostic nanoparticle (TNP) for photodynamic therapy of atherosclerosis disease. A) Aqueous solution of TNPs were stable for months without precipitation ( $\mathrm{pH} 7.4$ ) (left), and visualized through MRI (middle) and fluorescence imaging (0.01 mg Fe per mL) (right). B) Cellular uptake and light-induced phototoxicity of the TNPs (i) Flow cytometic analysis of time-dependent accumulation of TNPs as confirmed by cell-associated fluorescence (0.1 mg Fe per $\mathrm{mL}$ ). (ii) Fluorescence microscopy image shows an uptake and intracellular localization of the TNPs after $3 \mathrm{~h}$ incubation with RAW 264.7 cells ( $0.1 \mathrm{mg}$ Fe per $\mathrm{mL}$ ). (iii) Cell viability of human macrophages after incubation $(1 \mathrm{~h})$ with the respective nanoparticles $(0.2 \mathrm{mg} \mathrm{Fe} \mathrm{per} \mathrm{mL})$ and light treatment $\left(42 \mathrm{~mW} \mathrm{~cm}{ }^{-} 2,7.5 \mathrm{~J}\right)$, as determined by a MTS assay. The TNP Dark experiment consisted of cells incubated with TNP that did not receive PDT treatment. Control cells were incubated with PBS. All results are relative to the control cells ( $100 \%)$. (iv) Dose-dependent phototoxicity of TNP in RAW 264.7 cells after incubation ( 1 h) and light treatment ( 42 $\mathrm{mW} \mathrm{cm}-2,7.5 \mathrm{~J}$ ), as determined by the MTS assay. Reproduced with permission from[27]. 
with an average size of $18 \mathrm{~nm}$ and $\mathrm{pH}$ ( 5-8.0)-dependent zeta potential $(10-40 \mathrm{mV})$ [36]. The injected ALA/AuNPs accumulated into atheromatous plaques in a rabbit model of atherosclerosis and their ALA was converted to PPIX. The isolated blood and feces of rabbit demonstrated an increase in the fluorescence intensity of porphyrin, indicating stable encapsulation of ALA into gold nanoparticles and further conversion to fluorescent PPIX upon photo-exposure [36].

SIGNR1 is a principal receptor for uptake of microbial dextran polysaccharides by macrophages $[28,37]$. To target macrophages through the dextran receptor, near infrared (NIR) light-responsive dextran conjugated hollow-type gold nanoparticles were synthesized with an average size of $60 \mathrm{~nm}$ and $5 \mathrm{~nm}$ shell thickness; and these NPs were studied for their efficacy as a theranostic agent in RAW 267.4 macrophages [38]. The NPs were uptaken by macrophages through a phagocytosis mechanism, which were imaged by dark field microscopy. The uptaken NPs by macrophages were then exposed to a different dose of NIR light; and cell death was analyzed by flow cytometry. No cell death was observed for control conditions but exposed particles caused a particle dose-dependent death of macrophages either through late apoptosis or secondary necrosis in in vitro cell culture studies [38].

Activated macrophages express CD44 as one of cell surface receptors, and are known to phagocytose $[39,40]$, which provides an opportunity for macrophage targeting through extracellular hyaluronates. To target macrophages through a hyaluronate receptor, Frédéric et al. used hyaluronate-decorated chitosan nanogels embedded with anionic photosensitizer for photo-thermal therapy of inflamed macrophage rich region [41]. Ce6-hyaluronate-chitosan nanogels were formulated in a size range between 40 and $140 \mathrm{~nm}$, when measured by SEM, and exhibited a zeta potential of $-41 \mathrm{mV}$. Ce6-nanogels were preferentially uptaken either by murine RAW 264.7 or human THP-1 macrophages but negligibly uptaken by murine fibroblasts. When loaded in nanogels, photosensitizers retained in the inflamed joints of rheumatoid arthritis mice over a longer period of time compared to the free photosensitizers. This photodynamic therapy also exhibited a reduction in macrophage induced inflammation compared to the control conditions [41].

Another recent study by Qin et al. used gold nanorods (Au NRs) with dimension of $60 \times 14 \mathrm{~nm}$ as a promising theranostic approach for local imaging and photo-thermal therapy of inflamed macrophages [42] (Fig. 3). Au NRs were rigorously characterized for their efficacy as a nanotheranostic vehicle through in vitro experiments such as CCK8 cytotoxicity assay, calcein AM/PI staining, their cellular uptake by flow cytometry, CT imaging, and photo-thermal ablation treatment. Silver staining and in vitro micro-CT imaging demonstrated a concentration dependent uptake of AuNRs by macrophages. Next, a femoral artery restenosis model in apolipoprotein $\mathrm{E}$ knockout (Apo E) mice was used to examine the performance of $\mathrm{Au}$ NRs. After photo-thermal therapy (PTT), histological examination of femoral arteries staining images confirmed an ablation of macrophage layer in $\mathrm{Au}$ NRs group compared to the controls [42].

The uptake of USPIO by macrophages is widely used for non-invasive MRI assessment of inflammation in atherosclerotic plaques [43]. To study the effect of Angiotensin II (Ang II) infusion on chronic anti-inflammatory response, Morris et al. studied uptake of USPIO as a contrast agent for imaging of atherosclerotic plaque inflammation in aorta of apoE $\mathrm{E}^{-/-}$mice [44]. Histological examination of the aortic root lesion area after SB-239063 treatment indicated co-localization of USPIO inside the macrophages. Their study also demonstrated a reduction in uptake of USPIO by inflamed macrophages in atherosclerotic plaque after an anti-inflammatory treatment with p38 MAPK inhibitor[44].

To effectively target the collagen IV rich atherosclerosis plaques, Dong et al. prepared polymer-lipid hybrid nanoparticles embedded with ultra-small super paramagnetic iron oxide (USPIO) and paclitaxel and functionalized the particles with collagen targeting specific C11 polypeptide (UP-NP-C11) [45]. NPs with USPIO and paclitaxel were formulated through a nanoprecipitation method and showed an average diameter of $140 \mathrm{~nm}$. UP-NP-C11 NPs demonstrated sustained and control release behavior of paclitaxel. To demonstrate the targeting ability of C11 polypeptide in an in vitro study, macrophages coated over collagen IV rich Matrigel demonstrated a significant higher accumulation of UP-NP-C11 NPs compared to the control UP-NP NPs. Therefore, UP-NP-C11 NPs exhibited a better growth inhibitory effect on macrophages and good MRI ability. In vivo studies further confirmed the significantly higher accumulation of UP-NP-C11 NPs in rabbit atherosclerotic plaques compared to the control conditions [45].

In another approach, Dongjin et al. developed pH-responsive, doxorubicin (Dox)-loaded hyaluronic acid-polypyrrole nanoparticles (HA-PPyNPs) for fluorescence imaging and therapy of proliferating macrophages [46]. HA-PPyNPs were able to stably 
maintain the fluorescence quenching in a high serum (50\%) environment. HA-PPyNPs under acidic conditions were able to demonstrate the sustained release of Dox as compared to the physiological conditions. Next, HA-PPyNPs were utilized for in vitro live cell imaging to see the $\mathrm{pH}$ activated fluorescence imaging and release of DOX@HA-PPyNPs to macrophages. An effective uptake of HA-PPyNPs was demonstrated through an increase in fluorescence intensity inside the cytoplasm through $\mathrm{pH}$ triggered release of drug. The control HA-PPyNPs were cytocompatible but Dox loaded HA-PPyNPs exhibited significant toxicity to the raw macrophages. hVSMCs as control showed 90\% viability with Dox loaded HA-PPyNPs but 60\% macrophages were dead under similar treatment conditions indicated their selective targetability of NPs towards the macrophages. These results were mainly focused on in vitro conditions [46].
Duivenvoorden et al. from the Mulder group recently developed a statin loaded high density lipoprotein (rHDL)-based nanoparticles for delivery of statins at atherosclerotic lesions [47]. Statin is classified as a 3-hydroxy-3-methylglutaryl coenzyme A reductase (HMGR) inhibitor which is widely prescribed as a serum LDL cholesterol-lowering drug with anti-inflammatory properties. However, their full benefits cannot be harnessed due to low systemic availability. In vitro, selective anti-inflammatory effects of statin-rHDL nanoparticles on macrophages were demonstrated through very low cell viability of macrophages compared to smooth muscle cells and hepatocytes under similar culture conditions for 48 hours. When an apolipoprotein E-knockout mouse model of atherosclerosis was used, in vivo delivery of statin-rHDL nanoparticles was successfully demonstrated at the sites of atherosclerosis lesion and reduced the inflammation through targeting macrophages [47].
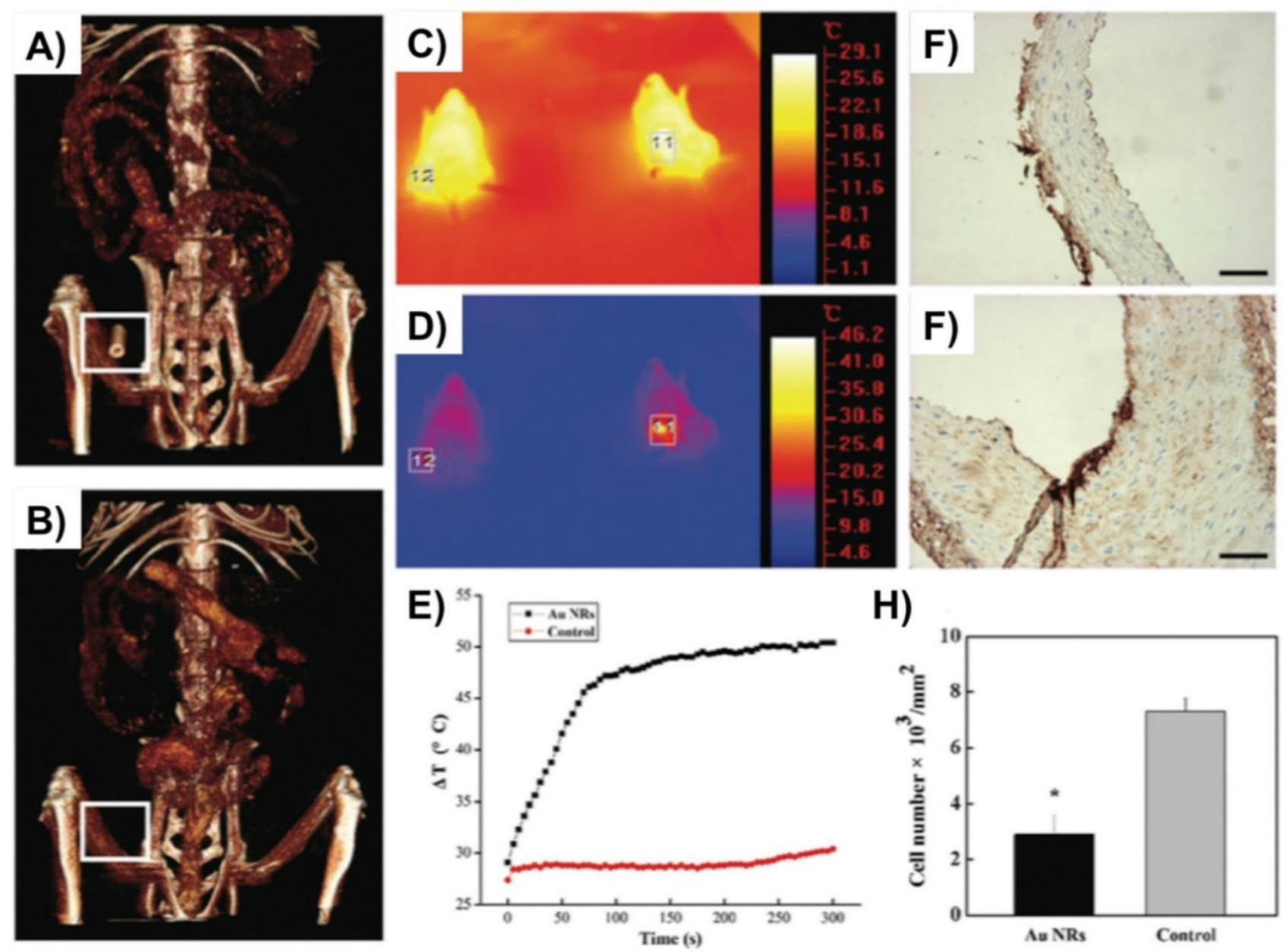

H)

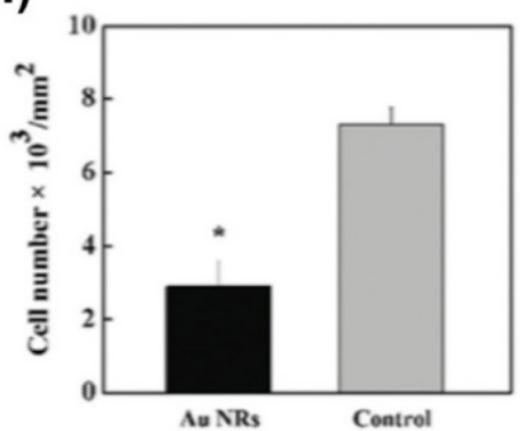

Figure 3. Gold nanorods (Au NRs) as a theranostic platform for imaging and photothermal therapy (PTT) of inflamed macrophages in a mouse model of femoral artery restenosis. (A, B) In vivo 3D micro-CT images of the femoral artery before (down; B) and after (up; A) intravenous injection of Au NRs. C, D) IR thermal images of Apo E mice injected with the Au NRs (the right mouse, indicated region 11) or saline (the left mouse, indicated region 12) via intravenous injection, respectively, irradiated with an $808 \mathrm{~nm}$ laser $\left(2 \mathrm{~W} / \mathrm{cm}^{2}\right.$ ) at a time point of 0 (up; C) and $300 \mathrm{~s}$ (down; C). E) The temperature profiles in regions 11 and 12 as a function of irradiation time. F, G) The representative histological examination sections stained with CD68, of the corresponding ex vivo femoral artery restenosis regions, after irradiation for $10 \mathrm{~min}$. Irradiation was done by an $808 \mathrm{~nm}$ laser with the safe power density of $2 \mathrm{~W} / \mathrm{cm}^{2}$. H) Statistical analysis of the macrophage number between Au NRs with and without PTT. Scale bars indicate $100 \mu \mathrm{m}$. Reproduced with permission from [42]. 
Vasa vasorum growth-mediated angiogenesis plays a crucial role in progression of atherosclerotic plaques and several tumor types [48]. To target endothelial cells through $\alpha_{v} \beta_{3}$ Integrin receptors, Winter et al. intravenously injected the $\alpha_{v} \beta_{3}$-targeting nanoparticles into a hyperlipidemic white rabbit model for non-invasive MRI imaging of molecular epitopes associated with angiogenesis [49]. Their follow-up study was designed to deliver anti-angiogenic drugs that could reduce or stop plaque progression and also allow for non-invasive assessment of intramural effectiveness of the treatment. $\operatorname{av} \beta 3$ integrin-targeted paramagnetic nanoparticles were loaded with an anti-angiogenic drug, fumagillin, and their efficacy was tested for site-specific, non-invasive, treatment of angiogenesis at an early stage of atherosclerosis [50]. NPs delivered the drug successfully and showed an anti-angiogenic response compared to controls, which was assessed through follow-up imaging of nanoparticle-accumulated areas. Adventitial angiogenic expansion of the vasa vasorum was also examined through microscopically and demonstrated a significant reduction in angiogenesis after introducing a first dose of $\alpha_{v} \beta_{3}$-targeted, fumagillin-loaded NPs in comparison to control conditions [50].

To explore the use of nucleoside-lipid based solid-lipid nanoparticle (SLNs) as a theranostic for potential prevention of platelet activation and aggregation at the site of atherosclerosis site, Khalid et al. formulated SLNs through encapsulation of USPIO and drug (a-tocopherol or prostacyclin PGI2) either with positively (DiC16dT) or negatively charged (DOTAU, DOU- polyethylene glycol (PEG)) lipids [51]. A nanoprecipitation method was used for preparation of positively or negatively charged SLNs with an average size of $80(\zeta=+55)$ and $98(\zeta-27 \mathrm{mV})$ $\mathrm{nm}$, respectively. SLNs showed colloidal stability at $37^{\circ} \mathrm{C}$ for 2 days without any change in their particle diameter. Magnetic resonance relaxometry measurement of SLNs demonstrated better concentration-dependent relaxivity properties compared to the clinically used Feridex as a contrast agent. To achieve long circulation time, negatively-charged SLNs were pegylated through mixing between nucleo-lipids DOTAU, and DOU-PEG and loaded with prostacyclin (PGI2) drug. Platelet aggregation induced by adenosine 5'-diphosphate (ADP) and thrombin receptor-activating peptide- 6 (TRAP-6) at post $15 \mathrm{~min}$ and $3 \mathrm{~h}$ incubation was completely inhibited by PEGylated SLNs loaded with USPIO and prostacyclin PGI2, whereas SLNs without prostacyclin PGI2 allowed full aggregation [51].
To selectively target atheroprone vasculature, Hofmeister et al. from the Sung and Harrison group used PREY peptide-conjugated DPHE liposomes as targeting nanoparticles (Fig. 4). PREY-targeted liposomes with an average hydrodynamic diameter of $64 \mathrm{~nm}$ accumulated preferentially in endothelial cells in a partially occluded carotid artery and other areas of disturbed flow [52]. Proteomic analysis and immunoblotting indicated that fibronectin and Filamin A were preferentially bound by PREY-nanocarriers in vessels under disturbed flow. In additional experiments, PREY-nanocarriers were loaded with the nitric oxide synthase co-factor tetrahydrobiopterin $\left(\mathrm{BH}_{4}\right)$ that was previously shown to be deficient in regions of disturbed flow [53]. This intervention increased vascular $\mathrm{BH}_{4}$ and reduced the vascular superoxide in the partially ligated artery in a wild-type mouse, reducing plaque burden in the partially ligated left carotid artery of fat-fed atheroprone mice $\left(\mathrm{ApoE}^{-/-}\right)$. This approach provides a potential means to prevent the formation of atherosclerotic lesions [52].

\section{Nanoparticle targeting strategies for Stenosis}

Stenosis is mainly caused by luminal narrowing of blood vessels due to buildup of atherosclerosis plaques inside arterial walls [54]. To treat stenosis, balloon dilation and expandable stent placement are being used as safe, minimally-invasive, and effective treatment options, but over time restenosis of treated arteries leads to adverse events such as myocardial infarction and blocked peripheral circulation [54].

For treatment of stenosis post balloon injury without delaying healing of damaged endothelial cells, Cyrus et al. used av $\beta 3$-targeted Rapamycin-loaded paramagnetic theranostic nanoparticles [55], which resulted in sustained and controlled release of anti-restenotic rapamycin in vitro. Restenosis was induced in a rabbit femoral artery model through feeding a high-cholesterol diet for 4 months. Magnetic resonance angiography images post two week treatment revealed that injured vessels treated with theranostic rapamycin-loaded NPs had less plaque formation and lumen occlusion than the control conditions [55].

In another study, to control the dose and release kinetics of paclitaxel from a magnetizable stent, Chorny et al. used polylactide-based, paclitaxel-loaded magnetic nanoparticles (PTX-MNPs) under uniform-field-induced magnetization for treatment of in-stent restenosis[56]. DLS-based measurement showed the MNPs had an average hydrodynamic diameter of $263 \mathrm{~nm}$ and a -12 $\mathrm{mV}$ zeta potential. Growth of arterial smooth muscle cells treated with PTX-loaded MNPs was significantly 
inhibited compared to the control in a nonmagnetic condition. A rat carotid artery model of in-stent restenosis was used for local delivery of clinically relevant MNP dose under uniform magnetic field, which demonstrated accumulation of significantly higher MNP amount to the stent and arterial tissue than the nonmagnetic control. This approach provided a site-specific drug delivery method to implanted magnetizable stents by uniform magnetic field-controlled targeting of MNPs for the treatment of in-stent restenosis [56].

\section{Nanoparticle targeting strategies for Peripheral Artery Disease}

PAD is a common type of vascular disease which is characterized by narrowing of peripheral arteries through buildup of plaques commonly seen in lower extremities, which reduces blood supply to head, organs and limbs. Therapeutic angiogenesis is identified as a treatment option for PAD through formation of new blood vessels by sprouting and branching of existing blood vessels in ischemic tissues
[57]. Successful angiogenesis is heavily dependent on effective and sustained delivery of VEGF to an ischemic site. Clinical administration of growth factors via bolus injection into either an ischemic site or intravenously was employed to induce therapeutic angiogenesis but failed to achieve significant therapeutic effects, primarily due to poor circulation time with the lack of targeting mechanism and sustained delivery of growth factors [58].

Sun et al. developed VEGF-loaded, IR800-conjugated, graphene oxide (GO-IR800-VEGF) NPs as a theranostic platform for imaging therapeutic angiogenesis of ischemic muscle in a mice hind limb model [59]. NPs were able to stably deliver a sufficient concentration of VEGF to an injury site. The efficacy of GO-IR800-VEGF to induce therapeutic angiogenesis of ischemic muscle was analyzed by LDI PA, and PET as imaging methods, which were further validated by ex vivo immunostaining of CD31 and a-SMA [59].
A)

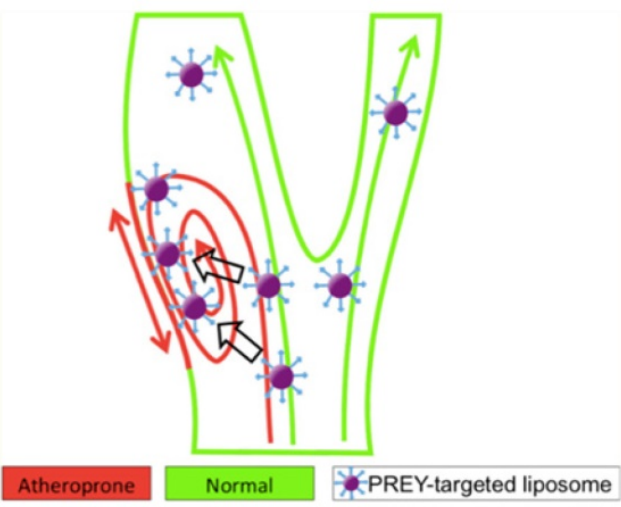

C)

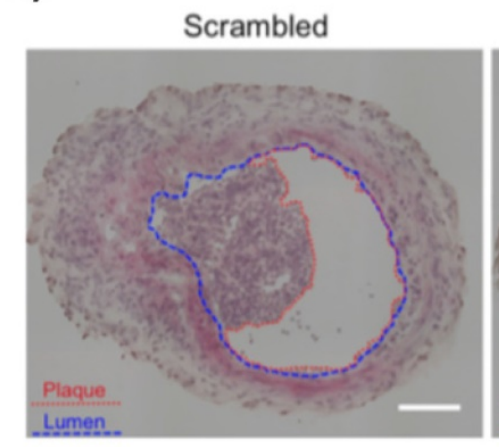

Targeted

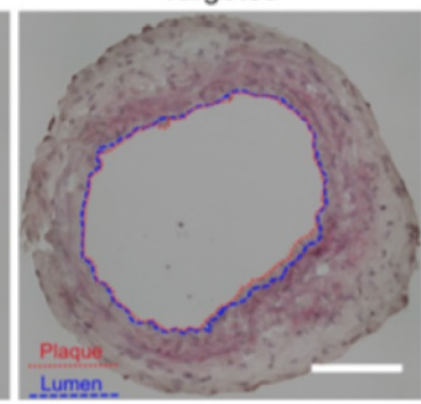

B)

(i)

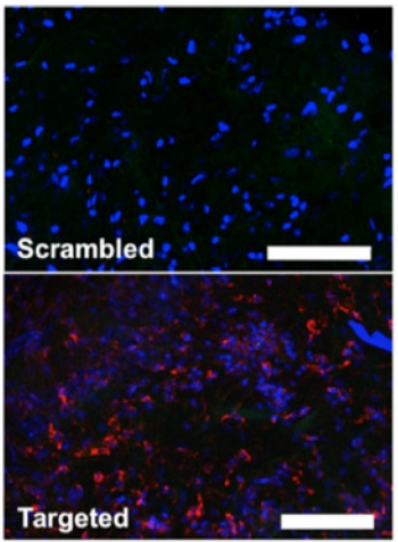

(ii)

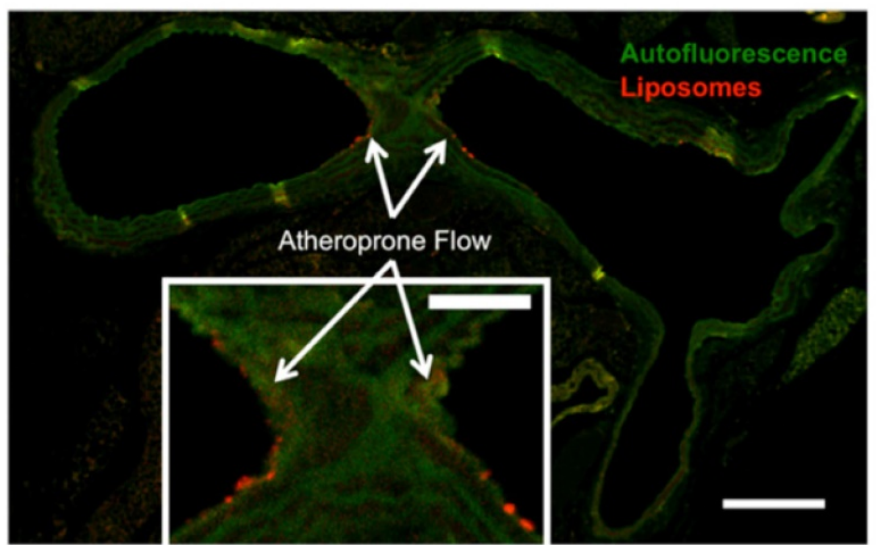

Figure 4. PREY(GSPREYTSYMPH)-targeted liposome for selectively targeting atheroprone vasculature. A) Ahterosclerosis preferentially develops in the regions of disturbed blood flow. PREY peptide (GSPREYTSYMPH) of nanocarrier was selectively accumulated in the luminal surface of endothelial cells caused by the atheroprone flow. B) Vascular targeting of PREY-nanocarriers. (i) En face preparations of ligated left carotid arteries with scrambled and targeted liposome injections (Red $=$ liposome fluorescence, blue $=$ nuclei). (ii) Cross section of a mouse aortic arch showing targeted liposome accumulation in the lesser curvature of the aortic arch (inset) (green = elastin autofluorescence, red = liposome fluorescence; Scale bar $=250 \mu \mathrm{m}$, inset scale bar $=100 \mu \mathrm{m}$ ). C) Liposome delivery of tetrahydrobiopterin $\left(\mathrm{BH}_{4}\right)$ significantly reduced plaque burden in the ligated left carotid artery of ApoE-/- mice fed a high fat diet for 7 days. Plaque is outlined in red, and lumen is outlined in blue. Scale bars $=100 \mu \mathrm{m}$. Reproduced with permission from [52]. 
In another approach to target a site of PAD through an EPR effect, Kim et al. used rhodamine Blabeled, PEGylated R-SiNPs for sustained delivery of VEGF in a murine model of hind limb ischemia [60]. PEGylated NPs with an average diameter of $80 \mathrm{~nm}$ showed longer blood circulation time and preferentially accumulated into ischemic site in a uniform fashion as compared to the non-PEGylated si-NPs. VEGF loaded, PEGylated NPs demonstrated the most efficient targeting ability within a week post-ischemia induction [60].

\section{Nanoparticle targeting strategies for Thrombosis}

Thrombosis is an acute medical condition of vascular system which is defined by formation of abnormal clot inside the blood vessels and obstruction in blood flow of the circulatory system [61]. It happens through deposition of platelets and fibrin as a form of clot to prevent blood loss from injured blood vessels. A ruptured clot from an injured blood vessel travels through the circulation system; struck somewhere; and thus damaged tissue and organ substantially [61]. Resorting the anti-thrombotic surface of affected vessels is promising treatment option for thrombosis [62]. High, specific affinities of D-phenylalanyl-L-prolyl-L-arginyl-chloromethyl

ketone (PPACK) and bivalirudin to thrombin are clinically proven but rapid in vivo clearance limits their clinical application as an anti-coagulant agent $[63,64]$. Generation of effective and long-lasting anticoagulant surface over a newly forming clot through the use of thrombin-targeted NPs provided another site-specific approach for management of acute thrombosis [64].

In order to form an anticlotting surface at the site of acute thrombosis, Myerson et al. developed either PACK or bivalirudin-functionalized perfluorocarbon NPs (PFC-NPs) as a thrombin-targeting theranostic platform. They demonstrated binding of PFC-NPs onto a site of active clotting, thereby quenching the local thrombin activity and inhibiting platelet deposition while exhibiting only a transient systemic anticoagulant effect [65]. A Fibrinopeptide A (FPA) ELISA assay confirmed binding of PPACK and bivalirudin to thrombin in free as well as when attached onto NPs. Thrombin-inhibiting NPS were injected into C57BL6 mice post inducing a laser injury of the carotid artery. A ${ }^{19} \mathrm{~F}-$ based MRI signal confirmed uniform accumulation of PFC-NPs in arterial thrombi. The specific binding and retention of thrombin-inhibiting NPs in injured arteries were verified by quantitative ${ }^{19} \mathrm{~F}$ spectroscopy [65].

In a similar manner, Palekar et al. conjugated PPACK as an anti-thrombotic agent onto the surface of liposomes and formulated it as single unilamellar vesicles with an average diameter of $170 \mathrm{~nm}$ having a nearly neutrally-charged surface [66]. The binding and anti-coagulant activity of vesicles was demonstrated through in vitro clot growth assays and fluorescent imaging. The in vivo efficacy of injected PPACK-liposomes as anti-coagulation agent was demonstrated by prolonged arterial occlusion time compared to controls in a mouse model of photochemical-induced carotid artery injury [66].

\section{Nanoparticle targeting strategies for Restenosis}

Restenosis mainly occurs due to remodeling of arterial wall through activation, proliferation and migration of vascular smooth muscle cells (VSMCs), and over production of extracellular matrix after balloon-angioplasty [67]. Since activated VSMCs express a higher level of tissue factor (TF) at a balloon injury site, Lanza et al. used TF-targeted NPs and identified angioplasty-induced expression of TF by SMCs within tunica media in a porcine carotid arteries model [68]. In their fellow-up study, TF-targeted biotinylated perfluorocarbon NPs were used as a theranostic platform for co-delivery of hydrophilic doxorubicin and hydrophobic paclitaxel to prevent restenosis since they are potent lipophilic anti-proliferative agents [69]. Both drugs-loaded NPs were able to successfully reduce VSMC proliferation compared to the treated control condition. Specific adherence of TF-targeted paclitaxel NPs with a large payload of paramagnetic chelates was visualized by T1-weighted ${ }^{1} \mathrm{H}$ MRI and detected by $19 \mathrm{~F}$ spectroscopy at 4.7T. However, the theranostic function was not confirmed in animal models [69].

\section{Nanoparticle targeting strategies for Aortic Aneurysms}

Aortic aneurysm (AA) is characterized by formation of a balloon-like bulge inside either the abdominal or thoracic aorta. It mainly occurs through vessel enlargement resulting from chronic inflammation which causes thinning and weakening of all three layers of the vessel wall [70]. Arterial walls weaken through calcification and MMP-induced degradation of vessel ECM. A survival rate following raptured AA balloon was ranged merely between $10-15 \%[70]$. To remove the mineral deposit and further restore the elastic lamina through therapeutic treatment, Nosoudi et al. developed EDTA- and PGG-loaded NPs with a particle size of around 200 $\mathrm{nm}$ and $\sim 39 \mathrm{mV} \zeta$ - potential as a dual therapeutic platform in a rat model of abdominal aortic aneurysms [71]. First, EDTA-loaded NPs were delivered to reduce calcification by removing calcium 
chloride in a vessel injury model, followed by delivery of pentagalloyl glucose (PGG)-loaded NPs, resulting in a reduction in development of calcified aneurysm. As a result, macrophage-induced inflammation was reduced with consequent decreases in calcification, elastin degradation, and MMP activity [71].

These research examples do not cover all the recent advances but provide necessary insights into the representative efforts done thus far in developing nanotheranostic platforms applied as vascular disease treatments. The common concept to design theranostic nanoparticles enables physicians to diagnose and treat vascular diseases simultaneously, thereby optimizing drug doses for the individual patient in a more efficient manner. These efforts will serve as a cornerstone to improve therapeutic and cost benefits while reducing adverse side effects $[16,17$, 72].

\section{Current limitations and future outlook}

The current review article highlights recent approaches in the development of theranostic NPs to enable real-time diagnosis and treatment of vascular diseases. Continuing advances in the fields of vascular medicine and nanotechnology enables the design and utilization of multifunctional NPs for effective monitoring of inflammation-related dysfunctional changes in endothelium, target-specific drug release to lesions, and healing processes such as retardation of atherosclerotic plaques or induction of thrombolysis. In vitro and in vivo experiments carried out in animal models and some preliminary pilot studies in humans exhibit promise in achieving these objectives [73].

Despite recent advances in the development of vascular nanotheranostics, there is no commercialized product. Main roadblocks include low sensitivity of MRI agents (e.g. iron oxide NPs); cost effectiveness (gold NPs), complex synthesis, long-term toxicity of nanomaterials used; and a lack of human disease models [74]. The vast majority of studies have been conducted in small animal models that have significantly different anatomies of vascular disease compared to humans [16]. Although, nanoscale size and charge allow for effective internalization of nanoparticles into cellular compartments, excessive accumulation of particles into tissue also poses toxicity risks. Therefore, extensive, prudent toxicological and pharmacological studies are necessary before considering clinical translation of NPs [75].

Translation from the bench to clinical research may be accelerated through tethering of biological drugs such as peptide and nucleic acids to theranostics and/or application of ex vivo human models in combination with organ-on-a-chip technologies, human induced pluripotent stem cells (iPSCs), and high-throughput screening techniques. Collaborative efforts between nanomaterial engineers, basic scientists, and clinicians are critical towards implementing and translating these technologies to achieve positive clinical outcomes.

\section{Acknowledgments}

This research was funded and supported by NSF CBET BME 1056046, NSF DMR BMAT 1506717, NIH EB 019509 and AHA GRNT25890018. This study was also financially supported by the Faculty Research Assistance Program of Yonsei University College of Medicine for 2016 (6-2016-0031). This study was also supported by Basic Science Research Program through the National Research Foundation of Korea (NRF) funded by the Ministry of Science, ICT \& Future Planning (2015R1C1A1A01051907)

\section{Competing Interests}

The authors have declared that no competing interest exists.

\section{References}

1. Mozaffarian D, Benjamin EJ, Go AS, Arnett DK, Blaha MJ, Cushman M, et al. Heart disease and stroke statistics-2015 update: a report from the american heart association. Circulation. 2015; 131: e29-e322.

2. Mozaffarian D, Benjamin EJ, Go AS, Arnett DK, Blaha MJ, Cushman M, et al. Heart Disease and Stroke Statistics-2016 Update. A Report From the American Heart Association. 2015.

3. Phillips DR, Conley PB, Sinha U, Andre P. Therapeutic approaches in arterial thrombosis. Journal of Thrombosis and Haemostasis. 2005; 3: 1577-89.

4. Libby P. The Forgotten Majority: Unfinished Business in Cardiovascular Risk Reduction. Journal of the American College of Cardiology. 2005; 46: 1225-8

5. Yin R-X, Yang D-Z, Wu J-Z. Nanoparticle Drug- and Gene-eluting Stents for the Prevention and Treatment of Coronary Restenosis. Theranostics. 2014; 4: 175-200.

6. Chen IY, Wu JC. Cardiovascular Molecular Imaging: Focus on Clinical Translation. Circulation. 2011; 123: 425-43.

7. Nederkoorn PJ, Elgersma OEH, Mali WPTM, Eikelboom BC, Kappelle LJ, van der Graaf Y. Overestimation of carotid artery stenosis with magnetic resonance angiography compared with digital subtraction angiography. Journal of Vascular Surgery. 2002; 36: 806-13.

8. Tabas I, Glass CK. Anti-Inflammatory Therapy in Chronic Disease: Challenges and Opportunities. Science. 2013; 339: 166-72.

9. Petros RA, DeSimone JM. Strategies in the design of nanoparticles for therapeutic applications. Nat Rev Drug Discov. 2010; 9: 615-27.

10. Kratz JD, Chaddha A, Bhattacharjee S, Goonewardena SN. Atherosclerosis and Nanotechnology: Diagnostic and Therapeutic Applications. Cardiovascular Drugs and Therapy. 2016; 30: 33-9.

11. Siemann DW, Bibby MC, Dark GG, Dicker AP, Eskens FALM, Horsman MR, et al. Differentiation and Definition of Vascular-Targeted Therapies. Clinical Cancer Research. 2005; 11: 416-20.

12. Alexis F, Pridgen E, Molnar LK, Farokhzad OC. Factors Affecting the Clearance and Biodistribution of Polymeric Nanoparticles. Molecular Pharmaceutics. 2008; 5: 505-15.

13. Agyare E, Kandimalla K. Delivery of Polymeric Nanoparticles to Target Vascular Diseases. Journal of biomolecular research \& therapeutics. 2014; 3: S1-001.

14. Funkhouser J. Reinventing Pharma: The Theranostic Revolution. Curr Drug Discovery. 2002; 2: 17-9.

15. Luk BT, Zhang L. Current Advances in Polymer-Based Nanotheranostics for Cancer Treatment and Diagnosis. ACS Applied Materials \& Interfaces. 2014; 6 : 21859-73.

16. Tang J, Lobatto ME, Read JC, Mieszawska AJ, Fayad ZA, Mulder WJM. Nanomedical Theranostics in Cardiovascular Disease. Current Cardiovascular Imaging Reports. 2012; 5: 19-25.

17. Lammers $T$, Kiessling F, Hennink WE, Storm G. Nanotheranostics and Image-Guided Drug Delivery: Current Concepts and Future Directions. Molecular Pharmaceutics. 2010; 7: 1899-912. 
18. Wang Z, Niu G, Chen X. Polymeric Materials for Theranostic Applications. Pharmaceutical Research. 2014; 31: 1358-76.

19. Jo SD, Ku SH, Won Y-Y, Kim SH, Kwon IC. Targeted Nanotheranostics for Future Personalized Medicine: Recent Progress in Cancer Therapy. Theranostics. 2016; 6: 1362-77.

20. Wickline SA, Neubauer AM, Winter P, Caruthers S, Lanza G. Applications of Nanotechnology to Atherosclerosis, Thrombosis, and Vascular Biology. Arteriosclerosis, Thrombosis, and Vascular Biology. 2006; 26: 435-41.

21. Psarros C, Lee R, Margaritis M, Antoniades C. Nanomedicine for the prevention, treatment and imaging of atherosclerosis. Nanomedicine: Nanotechnology, Biology and Medicine. 2012; 8(Supplement 1): S59-S68.

22. Gupta AS. Nanomedicine approaches in vascular disease: a review. Nanomedicine: Nanotechnology, Biology and Medicine. 2011; 7: 763-79.

23. Lusis AJ. Atherosclerosis. Nature. 2000; 407: 233-41.

24. Allahverdian S, Chehroudi AC, McManus BM, Abraham T, Francis GA. Contribution of intimal smooth muscle cells to cholesterol accumulation and macrophage-like cells in human atherosclerosis. Circulation. 2014; 129: 1551-9.

25. Robbins CS, Hilgendorf I, Weber GF, Theurl I, Iwamoto Y, Figueiredo J-L, et al. Local proliferation dominates lesional macrophage accumulation in atherosclerosis. Nat Med. 2013; 19: 1166-72.

26. Mulder WJM, Jaffer FA, Fayad ZA, Nahrendorf M. Imaging and Nanomedicine in Inflammatory Atherosclerosis. Science Translational Medicine. 2014; 6: 239sr1-sr1.

27. McCarthy JR, Jaffer FA, Weissleder R. A Macrophage-Targeted Theranostic Nanoparticle for Biomedical Applications. Small. 2006; 2: 983-7.

28. McCarthy JR, Korngold E, Weissleder R, Jaffer FA. A Light-Activated Theranostic Nanoagent for Targeted Macrophage Ablation in Inflammatory Atherosclerosis. Small. 2010; 6: 2041-9.

29. Kim D-E, Kim J-Y, Schellingerhout D, Kim E-J, Kim HK, Lee S, et al. Protease Imaging of Human Atheromata Captures Molecular Information of Atherosclerosis, Complementing Anatomic Imaging. Arteriosclerosis, Thrombosis, and Vascular Biology. 2010; 30: 449-56.

30. Choi Y, Weissleder R, Tung C-H. Selective Antitumor Effect of Novel Protease-Mediated Photodynamic Agent. Cancer Research. 2006; 66: 7225-9.

31. Lee DK, Choi Y, Shon S-M, Schellingerhout D, Park JE, Kim D-E. Atorvastatin and clopidogrel interfere with photosensitization in vitro. Photochemical \& Photobiological Sciences. 2011; 10: 1587-92.

32. Shon S-M, Choi Y, Kim J-Y, Lee DK, Park J-Y, Schellingerhout D, et al. Photodynamic Therapy Using a Protease-Mediated Theranostic Agent Reduces Cathepsin-B Activity in Mouse Atheromata In Vivo. Arteriosclerosis, Thrombosis, and Vascular Biology. 2013; 33: 1360-5.

33. Wachowska M, Muchowicz A, Firczuk M, Gabrysiak M, Winiarska M, Wańczyk M, et al. Aminolevulinic Acid (ALA) as a Prodrug in Photodynamic Therapy of Cancer. Molecules. 2011; 16: 4140.

34. Kwon O-C, Yoon H-J, Kim K-H, Kim H-T, Yoon Y-H, Kim J-K. Fluorescence Kinetics of Protoporphyrin-IX Induced from 5-ALA Compounds in Rabbit Postballoon Injury Model for ALA-Photoangioplasty. Photochemistry and Photobiology. 2008; 84: 1209-14.

35. Peng C, Li Y, Liang H, Cheng J, Li Q, Sun X, et al. Detection and photodynamic therapy of inflamed atherosclerotic plaques in the carotid artery of rabbits. Journal of Photochemistry and Photobiology B: Biology. 2011; 102: 26-31.

36. de Oliveira Goncalves K, da Silva MN, Sicchieri LB, de Oliveira Silva FR, de Matos RA, Courrol LC. Aminolevulinic acid with gold nanoparticles: a novel theranostic agent for atherosclerosis. Analyst. 2015; 140: 1974-80.

37. Taylor PR, Brown GD, Herre J, Williams DL, Willment JA, Gordon S. The Role of SIGNR1 and the $\beta$-Glucan Receptor (Dectin-1) in the Nonopsonic Recognition of Yeast by Specific Macrophages. The Journal of Immunology. 2004; 172: 1157-62

38. Yong Taik L, Mi Young C, Bang Sil C, Young-Woock N, Bong Hyun C. Diagnosis and therapy of macrophage cells using dextran-coated near-infrared responsive hollow-type gold nanoparticles. Nanotechnology. 2008; 19: 375105

39. Naor D, Nedvetzki S. CD44 in rheumatoid arthritis. Arthritis Res Ther. 2003; 5: 105

40. Vachon E, Martin R, Plumb J, Kwok V, Vandivier RW, Glogauer M, et al. CD44 is a phagocytic receptor. Blood. 2006; 107: 4149 .

41. Schmitt F, Lagopoulos L, Käuper P, Rossi N, Busso N, Barge J, et al. Chitosan-based nanogels for selective delivery of photosensitizers to macrophages and improved retention in and therapy of articular joints. Journal of Controlled Release. 2010; 144: 242-50.

42. Qin J, Peng Z, Li B, Ye K, Zhang Y, Yuan F, et al. Gold nanorods as a theranostic platform for in vitro and in vivo imaging and photothermal therapy of inflammatory macrophages. Nanoscale. 2015; 7: 13991-4001.

43. Schmitz SA, Taupitz M, Wagner S, Wolf K-J, Beyersdorff D, Hamm B. Magnetic resonance imaging of atherosclerotic plaques using superparamagnetic iron oxide particles. Journal of Magnetic Resonance Imaging. 2001; 14: 355-61.

44. Morris JB, Olzinski AR, Bernard RE, Aravindhan K, Mirabile RC, Boyce R, et al. p38 MAPK Inhibition Reduces Aortic Ultrasmall Superparamagnetic Iron Oxide Uptake in a Mouse Model of Atherosclerosis. MRI Assessment. 2008; 28: 265-71.

45. Dong $\mathrm{Y}$, Chen $\mathrm{H}$, Chen $\mathrm{C}$, Zhang $\mathrm{X}$, Tian $\mathrm{X}$, Zhang $\mathrm{Y}$, et al. Polymer-Lipid Hybrid Theranostic Nanoparticles Co-Delivering Ultrasmall Superparamagnetic Iron Oxide and Paclitaxel for Targeted Magnetic
Resonance Imaging and Therapy in Atherosclerotic Plaque. Journal of Biomedical Nanotechnology. 2016; 12: 1245-57.

46. Park D, Cho Y, Goh S-H, Choi Y. Hyaluronic acid-polypyrrole nanoparticles as pH-responsive theranostics. Chemical Communications. 2014; 50: 15014-7.

47. Duivenvoorden R, Tang J, Cormode DP, Mieszawska AJ, Izquierdo-Garcia D, Ozcan C, et al. A statin-loaded reconstituted high-density lipoprotein nanoparticle inhibits atherosclerotic plaque inflammation. Nature Communications. 2014; 5: 3065.

48. Pant S, Deshmukh A, Mehta JL. Angiogenesis in Atherosclerosis: An Overview. In: Mehta JL, Dhalla NS, editors. Biochemical Basis and Therapeutic Implications of Angiogenesis. New York, NY: Springer New York. 2013: 209-24.

49. Winter PM, Morawski AM, Caruthers SD, Fuhrhop RW, Zhang H, Williams TA, et al. Molecular imaging of angiogenesis in early-stage atherosclerosis with alpha(v)beta3-integrin-targeted nanoparticles. Circulation. 2003; 108: 2270-4.

50. Winter PM, Neubauer AM, Caruthers SD, Harris TD, Robertson JD, Williams TA, et al. Endothelial av $\beta 3$ Integrin-Targeted Fumagillin Nanoparticles Inhibit Angiogenesis in Atherosclerosis. Arteriosclerosis, Thrombosis, and Vascular Biology. 2006; 26: 2103-9.

51. Oumzil K, Ramin MA, Lorenzato C, Hémadou A, Laroche J, Jacobin-Valat MJ, et al. Solid Lipid Nanoparticles for Image-Guided Therapy of Atherosclerosis. Bioconjugate Chemistry. 2016; 27: 569-75.

52. Hofmeister LH, Lee SH, Norlander AE, Montaniel KRC, Chen W, Harrison DG, et al. Phage display-guided nanocarrier targeting to atheroprone vasculature. ACS nano. 2015; 9: 4435-46.

53. Landmesser U, Dikalov S, Price SR, McCann L, Fukai T, Holland SM, et al. Oxidation of tetrahydrobiopterin leads to uncoupling of endothelial cell nitric oxide synthase in hypertension. J Clin Invest. 2003; 111: 1201-9.

54. Otto CM, Prendergast B. Aortic-Valve Stenosis - From Patients at Risk to Severe Valve Obstruction. New England Journal of Medicine. 2014; 371: $744-56$.

55. Cyrus T, Zhang H, Allen JS, Williams TA, Hu G, Caruthers SD, et al. Intramural Delivery of Rapamycin With $\alpha(v) \beta(3)$-Targeted Paramagnetic Nanoparticles Inhibits Stenosis After Balloon Injury. Arteriosclerosis, Thrombosis, and Vascular Biology. 2008; 28: 820-6.

56. Chorny M, Fishbein I, Yellen BB, Alferiev IS, Bakay M, Ganta S, et al. Targeting stents with local delivery of paclitaxel-loaded magnetic nanoparticles using uniform fields. Proceedings of the National Academy of Sciences of the United States of America. 2010; 107: 8346-51.

57. Bhise NS, Shmueli RB, Sunshine JC, Tzeng SY, Green JJ. Drug delivery strategies for therapeutic angiogenesis and antiangiogenesis. Expert opinion on drug delivery. 2011; 8: 485-504.

58. Henry TD, Annex BH, McKendall GR, Azrin MA, Lopez JJ, Giordano FJ, et al. The VIVA Trial. Vascular Endothelial Growth Factor in Ischemia for Vascular Angiogenesis. 2003; 107: 1359-65.

59. Sun Z, Huang P, Tong G, Lin J, Jin A, Rong P, et al. VEGF-loaded graphene oxide as theranostics for multi-modality imaging-monitored targeting therapeutic angiogenesis of ischemic muscle. Nanoscale. 2013; 5: 6857-66.

60. Kim J, Cao L, Shvartsman D, Silva EA, Mooney DJ. Targeted Delivery of Nanoparticles to Ischemic Muscle for Imaging and Therapeutic Angiogenesis. Nano Letters. 2011: 11: 694-700.

61. Thompson AE. Deep vein thrombosis. JAMA. 2015; 313: 2090

62. Xu XR, Carrim N, Neves MAD, McKeown T, Stratton TW, Coelho RMP, et al. Platelets and platelet adhesion molecules: novel mechanisms of thrombosis and anti-thrombotic therapies. Thrombosis Journal. 2016; 14: 29.

63. Zamora R. Successful anticoagulation with bivalirudin in antithrombin-deficient pediatric patient undergoing stent placement. Catheterization and Cardiovascular Interventions. 2006; 68: 292-5.

64. Myerson J, He L, Lanza G, Tollefsen D, Wickline S. Thrombin-inhibiting perfluorocarbon nanoparticles provide a novel strategy for the treatment and manetic resonance imaging of acute thrombosis. Journal of Thrombosis and Haemostasis. 2011; 9: 1292-300.

65. Jacob Wheatley M, Li H, John Stacy A, Todd W, Gregory L, Douglas T, et al. Thrombin-inhibiting nanoparticles rapidly constitute versatile and detectable anticlotting surfaces. Nanotechnology. 2014; 25: 395101.

66. Palekar RU, Myerson JW, Schlesinger PH, Sadler JE, Pan H, Wickline SA. Thrombin-Targeted Liposomes Establish a Sustained Localized Anticlotting Barrier against Acute Thrombosis. Molecular Pharmaceutics. 2013; 10: 4168-75.

67. Buccheri D, Piraino D, Andolina G, Cortese B. Understanding and managing in-stent restenosis: a review of clinical data, from pathogenesis to treatment. Journal of Thoracic Disease. 2016; 8: E1150-E62.

68. Lanza GM, Abendschein DR, Hall CS, Scott MJ, Scherrer DE, Houseman A, et al. In Vivo Molecular Imaging of Stretch-Induced Tissue Factor in Carotid Arteries with Ligand-Targeted Nanoparticles. Journal of the American Society of Echocardiography. 2000; 13: 608-14

69. Lanza GM, Yu X, Winter PM, Abendschein DR, Karukstis KK, Scott MJ, et al. Targeted Antiproliferative Drug Delivery to Vascular Smooth Muscle Cells With a Magnetic Resonance Imaging Nanoparticle Contrast Agent. Implications for Rational Therapy of Restenosis. 2002; 106: 2842-7.

70. Kent KC. Abdominal Aortic Aneurysms. New England Journal of Medicine. 2014; 371: 2101-8.

71. Nosoudi N, Chowdhury A, Siclari S, Karamched S, Parasaram V, Parrish J, et al. Reversal of Vascular Calcification and Aneurysms in a Rat Model Using 
Dual Targeted Therapy with EDTA- and PGG-Loaded Nanoparticles. Theranostics. 2016; 6: 1975-87.

72. Mieszawska AJ, Mulder WJM, Fayad ZA, Cormode DP. Multifunctional Gold Nanoparticles for Diagnosis and Therapy of Disease. Molecular Pharmaceutics. 2013; 10: 831-47.

73. Fayad ZA, Mani V, Woodward M, Kallend D, Abt M, Burgess T, et al. Safety and efficacy of dalcetrapib on atherosclerotic disease using novel non-invasive multimodality imaging (dal-PLAQUE): a randomised clinical trial. The Lancet. 2011;378: 1547-59

74. Xie J, Lee S, Chen X. Nanoparticle-based theranostic agents. Advanced Drug Delivery Reviews. 2010; 62: 1064-79.

75. De Jong $\mathrm{WH}$, Borm PJA. Drug delivery and nanoparticles: Applications and hazards. International Journal of Nanomedicine. 2008; 3: 133-49. 